\title{
Photothermal detection of trace compounds in water, using the deflection of a water meniscus
}

\author{
Jane Hodgkinson ${ }^{\# *}{ }^{\mathrm{H}}$, Mark Johnson ${ }^{\#}$ and John P. Dakin*
}

\# North West Water Ltd, Dawson House, Great Sankey, Warrington WA5 3LW

* Optoelectronics Research Centre, Mountbatten Building, University of Southampton, Highfield, Southampton SO17 1BJ

H Now at: BG Technology, Gas R\&T Centre, Ashby Rd, Loughborough, Leics LE11 3GR

\begin{abstract}
A novel photothermal detector is described, based on a closed cell suitable for use with low-frequency modulated continuous-wave light sources. Photothermal expansion in aqueous samples caused the deflection of a water meniscus held across a $200 \mu \mathrm{m}$-radius pinhole. Displacement of the water meniscus was measured using fibre optic interferometry. A mercury discharge lamp $(254 \mathrm{~nm})$ and a laser diode $(678 \mathrm{~nm})$ were used to detect to absorption by $2 \mathrm{ppb}$ anthracene and $0.5 \mathrm{ppm}$ potassium permanganate in aqueous solution, respectively. The technique was used to detect differences between absorption coefficients in aqueous solutions down to approximately $20 \%$ of the background absorption of the water itself.
\end{abstract}

\section{Introduction}

The measurement of optical absorption in water is a well-known analytical technique. Photoacoustic detection of trace chemicals in water has a number of advantages over conventional transmission spectroscopy, chiefly a high signal to noise ratio and relative insensitivity to light scattering within the sample ${ }^{[1,2]}$.

Much recent work has been concerned with the detection of transient signals excited by high power pulsed light sources ${ }^{[3]}$. Hand et al have used a fibre-optic interferometer to measure the transient displacement of the surface of liquids caused by photoacoustic absorption of laser pulses in the bulk ${ }^{[4,5]}$. Their open cell design was appropriate for use with a pulsed source, but not suited to modulated cw sources.

Our technique has a number of similarities to the Golay cell, which was developed as a detector of infrared radiation ${ }^{[6,7]}$ but was later refined for photoacoustic gas detection ${ }^{[8]}$. Thermal expansion of the gas within the closed cell resulted in deflection of a thin, flexible mirror, detected with sub-Ångstrom sensitivity using an optical technique.

This paper addresses the operation of a novel photothermal system and its use in the detection of trace compounds in water. A mercury discharge lamp and a laser diode were used as low-cost, reliable light sources suitable for use within a process environment. These sources may be electronically modulated over a wide range of frequencies, avoiding the additional acoustic noise at the modulation frequency which would be expected when using a mechanical chopper. 


\section{Principle of operation}

A schematic diagram of the closed cell is shown in Figure 1. Light of a characteristic wavelength is absorbed by target chemicals, heating the solvent and causing it to expand. In a closed cell, this expansion of the fluid lying in the optical path increases the pressure on the surrounding fluid and on itself. The magnitude of the pressure increase is determined by the mechanical compressibility, or compliance $(\partial \mathrm{V} / \partial \mathrm{P})$, of the cell and the enclosed fluid. At the top of the cell a flat, horizontal water meniscus, constrained by surface forces across a $200 \mu \mathrm{m}$ pinhole, acts as a sensor. Pressure changes within the cell cause the centre of the meniscus to be deflected and this displacement is monitored using fibre optic interferometry. The magnitude of the photothermal response of the system has been considered in detail in the Appendix.

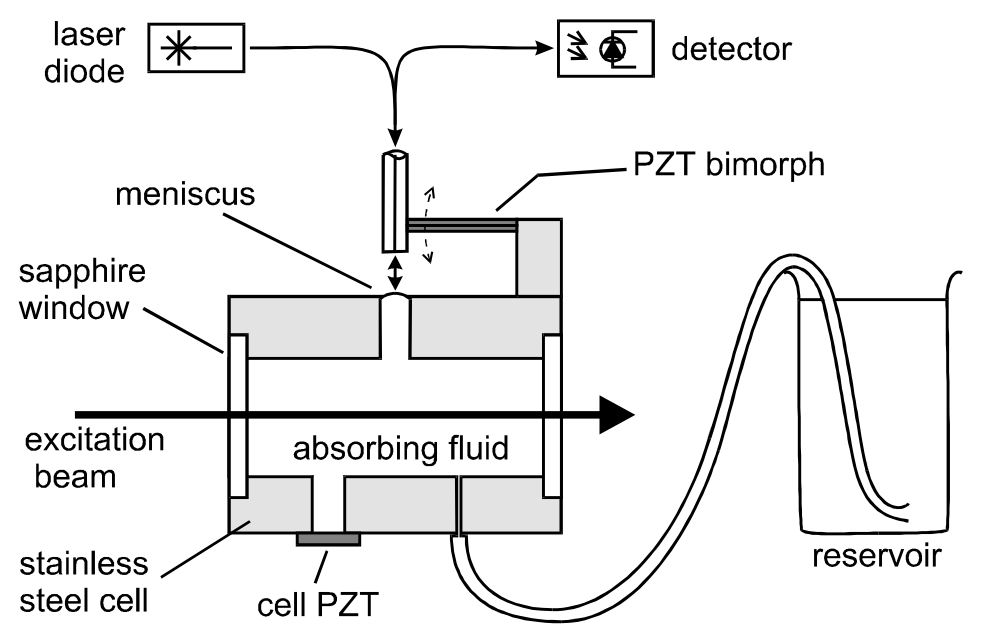

Figure 1. Schematic diagram of photothermal detector

Because of its similarity to an ordinary fluid-in-glass thermometer, we have described this arrangement as a photothermal detector. Moreau et al have found that the sensitivity of such thermometers is limited in practice by irregularities in the movement of the fluid meniscus caused by contact angle hysteresis at the fluid / glass interface ${ }^{[9]}$. In our technique, the problem of contact angle hysteresis has been removed by pinning the edges of the fluid meniscus at the pinhole. The curvature of the meniscus is then determined by the excess pressure in the cell. We have therefore created a novel pressure transducer and the apparatus could equally well be described as a low-frequency photoacoustic detector.

Anthracene (a polycyclic aromatic hydrocarbon, $\mathrm{PAH})$ and potassium permanganate $\left(\mathrm{KMnO}_{4}\right)$ were chosen as representative species to be detected in aqueous solution. PAHs are carcinogenic pollutants of interest to the water industry. $\mathrm{KMnO}_{4}$ is a known photoacoustic absorption standard which does not fluoresce, phosphoresce or undergo photochemical reactions when excited at $678 \mathrm{~nm}$, and therefore delivers all the absorbed energy quickly to the solvent as heat ${ }^{[10]}$.

\section{Experimental}

\subsection{Cell design}

The cell had a cylindrical internal bore of length $50 \mathrm{~mm}$ and radius $10 \mathrm{~mm}$. The inside of the cell was polished to increase its specular reflectivity and reduce the magnitude of the possible spurious signal resulting from scattered light hitting the cell walls. The sapphire windows were clamped against the metal end surfaces with a thin polymer sealing ring, so that they could easily be removed for cleaning. 
The meniscus was formed at a $200 \mu \mathrm{m}$ radius pinhole in a horizontal nickel foil disc, which was $3 \mathrm{~mm}$ in diameter and $40 \mu \mathrm{m}$ thick. A selection of pinhole diameters was available as a Utility Pinhole Set (Ealing Electro-Optics). Zero mean pressure difference across the meniscus was maintained by a slow hydraulic leak to an outside reservoir, which equalised internal and external pressures at frequencies much lower than the photothermal modulation frequency. This ensured that, on average, the meniscus was flat.

A piezoelectric element on the side of the cell was used to modulate the cell volume for test purposes (Morgan Matroc PZT-5A, 0.48mm thick, 10mm square, bonded to a $6 \mathrm{~mm}$ diameter hole in the cell wall). Applying a voltage across the cell PZT resulted in a large meniscus deflection, whose magnitude was proportional to the applied voltage.

\subsection{Interrogation of the meniscus by fibre optic interferometry}

The apparatus used to measure vertical meniscus deflection is shown in Figure 2. The interferometer cavity was formed by (a) the end of a cleaved optical fibre, and (b) the centre of the meniscus. In order to eliminate back-reflections from other fibre endfaces, fibre ferrules were polished at an angle of $12^{\circ}$ to the normal, and aligned at an angle of $6^{\circ}$ to the optical path ${ }^{[11]}$. The photodiode at (2) was connected to a transimpedance amplifier.

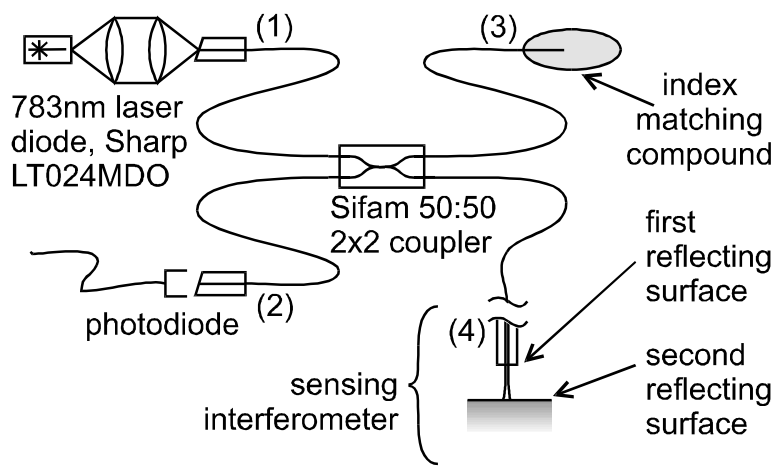

Figure 2. Low finesse fibre Fabry-Perot interferometer, used to detect the relative position of the meniscus

Phase quadrature was maintained using a piezoelectric bimorph element (Quantelec EB-T-320, 15mm $\times$ $1.5 \mathrm{~mm} \times 0.6 \mathrm{~mm}$ ) to change the vertical position of the cleaved end of the optical fibre (4). A slow feedback control loop, with a time constant of approximately $0.2 \mathrm{~s}$, applied a variable voltage to the bimorph in order to maintain a fixed voltage output from the detector, mechanically tracking an interference fringe edge. The voltage setpoint was adjusted manually at the beginning of each experiment. A distance of approximately $60 \mu \mathrm{m}$ was maintained between the fibre end and the meniscus to avoid accidental contact between the two.

\subsection{Acoustic isolation}

The noise floor for the interferometer was found to be below $1 \mathrm{pm} \mathrm{Hz}^{-1 / 2}$, measured with the cleaved fibre end over a silica test plate bonded next to the meniscus. However, the minimum detectable meniscus deflection was determined by environmental acoustic noise in the laboratory. An isolation chamber, shown in Figure 3, was used to reduce the level of acoustic noise transmitted through the air and through the ground / laboratory bench. The chamber reduced the magnitude of the noise voltage signals measured with the optical fibre / meniscus by a factor of approximately 100 , for frequencies between 100 and $500 \mathrm{~Hz}$. The resulting noise floor for the interferometer, in the region from $70 \mathrm{~Hz}$ to $120 \mathrm{~Hz}$, was approximately $10 \mathrm{pm} \mathrm{Hz}^{-1 / 2}$, which was believed to be determined by fluctuations of the meniscus rather than the fibre. 


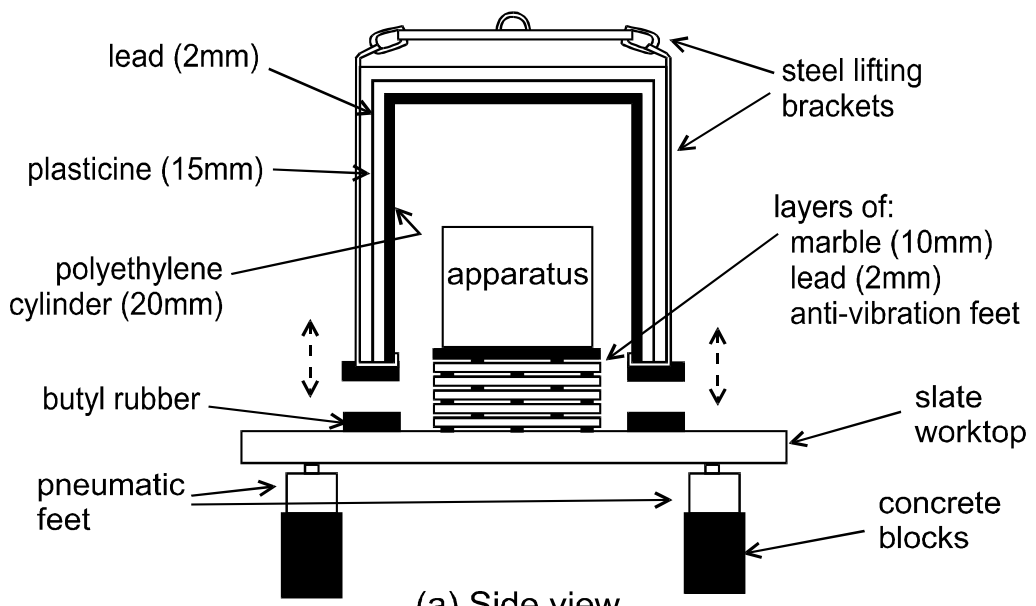

(a) Side view

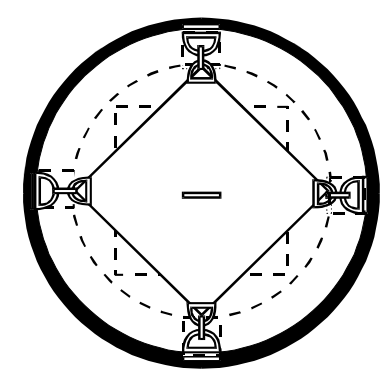

(b) Top view

Figure 3. Acoustic isolation chamber, used for photothermal experiments.

\subsection{System configuration}

Two light sources were used for our experiments. The first was a collimated $678 \mathrm{~nm}$ laser diode module (Vector Technology Beta TX, 3mW) with integral driver, which was modulated by applying a square-wave voltage from a signal generator (Thurlby Thandar model TG220).

The second light source was a mercury discharge lamp (Spectronics Spectroline 11SC-1), normally driven at mains frequency via its proprietary driver (Spectroline SCT 1A/F). Variable frequency operation was achieved by connecting the output from the signal generator to a power amplifier (ILP SMOS 248), and using a transformer (Intram Barwell MF100) to provide the $230 \mathrm{~V}$ required by the Spectroline lamp driver. $100 \%$ modulation was achieved for an applied sinusoidal voltage at frequencies of $\mathrm{f}>30 \mathrm{~Hz}$. The lamp intensity was modulated essentially as a rectified sine wave, the majority of the modulation occurring at $2 \mathrm{f}$. Stray light from the lamp was reduced by covering the mercury tube with aluminium foil, apart from a $3 \mathrm{~mm}$ aperture from which light was coupled into the cell using a high NA lens. A UV-enhanced photodiode was bonded over a second small hole in the foil and used to monitor the relative emission intensity from the lamp. A shutter was placed in front of the lamp, to allow it to warm up without exposing solutions in the cell to UV light, reducing the possibility of photobleaching.

The emitted powers of both light sources were estimated using a large area UV-enhanced silicon photodiode (Centronic OST100/7CQ). The photodiode current was related to the incident light intensity using the manufacturer's quoted responsivity at each wavelength, with an estimated absolute error of $15 \%$. The quoted responsivity at $780 \mathrm{~nm}$ was checked using a commercial fibre optic power meter (Megger OTP 510, also incorporating a silicon photodiode).

The UV lamp was modulated at a frequency of $\mathrm{f}=57.5 \mathrm{~Hz}(2 \mathrm{f}=115 \mathrm{~Hz})$. The $2 \mathrm{f}$-modulated $254 \mathrm{~nm}$ light power, transmitted into the cell, was estimated to be $0.30 \pm 0.05 \mathrm{~mW}$ rms. The output from the $678 \mathrm{~nm}$ laser diode was estimated to be $1.4 \pm 0.2 \mathrm{~mW} \mathrm{rms,} \mathrm{for} \mathrm{square} \mathrm{wave} \mathrm{modulation} \mathrm{at} 115 \mathrm{~Hz}$.

The configuration of the system is shown in Figure 4. Signals from the interferometer receiver were detected synchronously using a lock-in amplifier (EG\&G model $5210,0.5 \mathrm{~Hz}-110 \mathrm{kHz}$ ), in R, $\theta$ mode with a 100s time constant. For one series of experiments, the two light sources were used simultaneously. A second signal generator of the same type was used, with a lock-in amplifier which was similar to the first (EG\&G model 5110, $0.5 \mathrm{~Hz}-100 \mathrm{kHz}$ ), used in the same operating mode. 


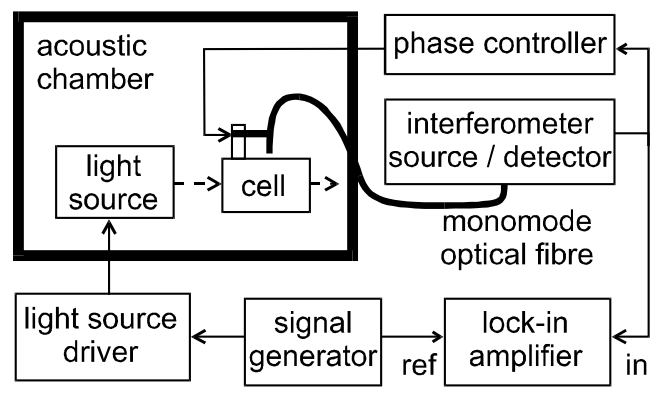

Figure 4. Schematic diagram of system components for photothermal detection.

\subsection{Experimental procedure}

The cell was filled with a test solution and the interferometer aligned by eye to the centre of the meniscus using an xyz stage. A sinusoidal voltage of approximately $10 \mathrm{~V}$ peak-to-peak was applied to the cell PZT, in order to modulate the cell volume and thereby the height of the centre of the meniscus. The resulting fringes from the interferometer were displayed on an oscilloscope and used to align the fibre more precisely, to the position of maximum meniscus movement. The interference fringes also were used to determine the correct quadrature setpoint for the phase controller.

Photoacoustic signals have a strong temperature dependence in water, which is approximately linear over our working range $\left(23-25^{\circ} \mathrm{C}\right)$, and falls to zero at $4^{\circ} \mathrm{C}^{[12]}$. The ambient temperature was measured for each result and used as a normalising factor. It was assumed that the $\mathrm{KMnO}_{4}$ solutions had reached thermal equilibrium with the surroundings, and that any temperature increase during each measurement was negligible. Also measured was the sinusoidal voltage required across the cell PZT to cause the meniscus to move by two whole interference fringes $(780 \mathrm{~nm})$. This voltage was inversely proportional to the responsivity of the cell to volume changes, and was used as a normalising factor to correct for possible changes in the surface tension of the fluid.

\section{$4 \quad$ Results and discussion}

The absorption spectra of aqueous solutions of anthracene and $\mathrm{KMnO}_{4}$, measured using the diode array spectrometer with a $4 \mathrm{~cm}$ pathlength cell, are shown in Figure 5 and Figure 6. The emission spectra of the laser diode and the mercury lamp, also measured using the spectrometer, have been superimposed. Many PAHs, including anthracene, are known to be unstable to high intensity UV light ${ }^{[13]}$, but no evidence of UV photolysis of our samples, which would have been evident as photobleaching, was observed during our experiments. The emission of the $678 \mathrm{~nm}$ laser diode corresponds to a region of relatively low absorption by the $\mathrm{KMnO}_{4}$. However, we were not primarily interested in detecting trace levels of $\mathrm{KMnO}_{4}$, but in characterising the system in terms of detectable absorption coefficients, otherwise a different wavelength might have been chosen. 


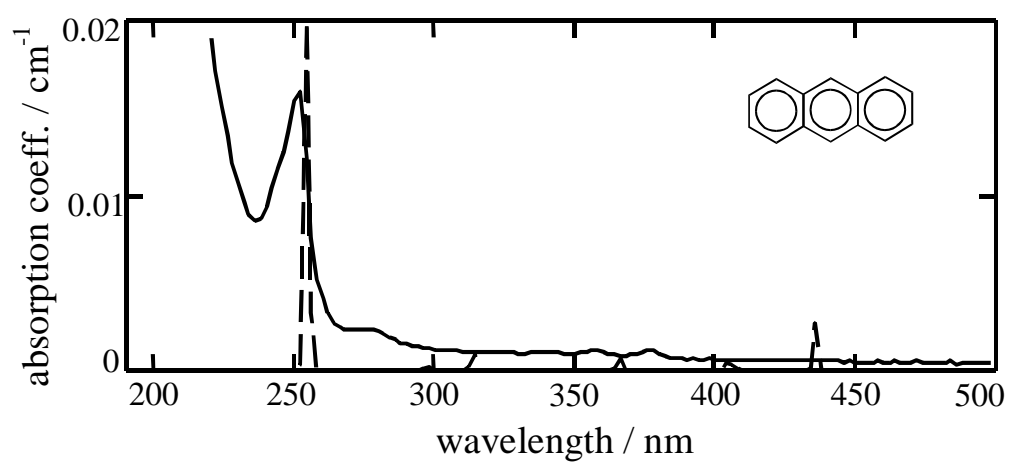

Figure 5. Absorption of anthracene (solid line) in aqueous solution, taken against a deionised water blank. The emission spectrum of the mercury lamp (dashed line, arbitrary units) has been superimposed.

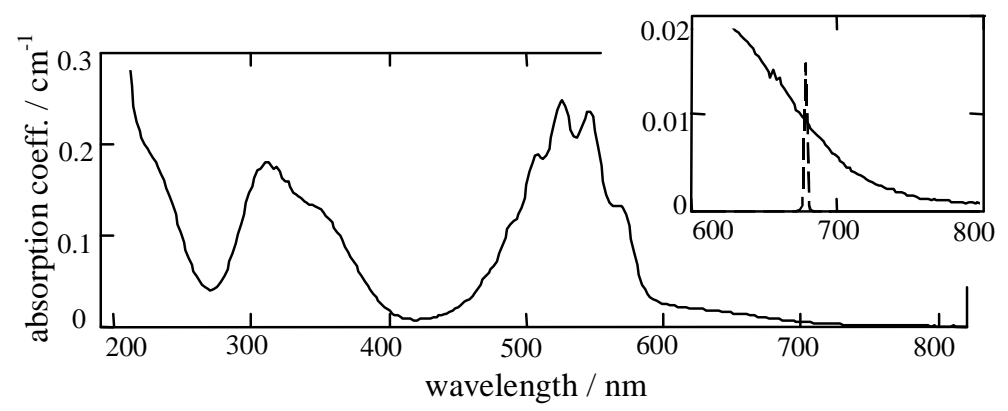

Figure 6. Absorption spectrum of $17 \mathrm{ppm} \mathrm{KMnO}_{4}$ in aqueous solution, taken against a deionised water blank. Inset: Expanded scale, with superimposed emission spectrum of the red laser diode (arbitrary units, dashed line).

Beer's law states that the absorbed light intensity is equal to

$$
I=I_{0}\left(1-10^{-\alpha \ell}\right)
$$

where $\mathrm{I}_{0}$ is the incident light intensity, $\ell$ is the pathlength in $\mathrm{cm}$, and $\alpha$ is absorption coefficient of the fluid, in $\mathrm{cm}^{-1}$. The absorption coefficients of different constituents of a solution are additive. At low levels of concentration (low $\alpha$ ) the intensity of absorbed light, and therefore the photothermal signal, is expected to be a linear function of the total absorption coefficient of the solution. The predicted responsivity of photothermal meniscus deflection is calculated in the Appendix, as is the expected magnitude of the resulting signal which would be detected by the interferometer receiver.

\subsection{Detection of potassium permanganate}

The relative concentrations of the $\mathrm{KMnO}_{4}$ samples were checked by measuring their absorption at $678 \mathrm{~nm}$, using the diode array spectrometer. The absorptions of the weakest solutions were too low for accurate measurements, so their concentrations were deduced from the measured value at $530 \mathrm{~nm}$, which corresponded to an absorption maximum.

The results for a range of solution concentrations are plotted in Figure 7 (a), versus the concentration of $\mathrm{KMnO}_{4}$ in ppm by weight. At high absorptions (approaching $\alpha=1$ ) the signals showed a non-linear response, as expected from Beer's law. At low levels of $\mathrm{KMnO}_{4}$, the signals reached a plateau corresponding to the background level of water absorption; the mean measured photothermal signal for deionised water control samples is shown as a dashed line. 


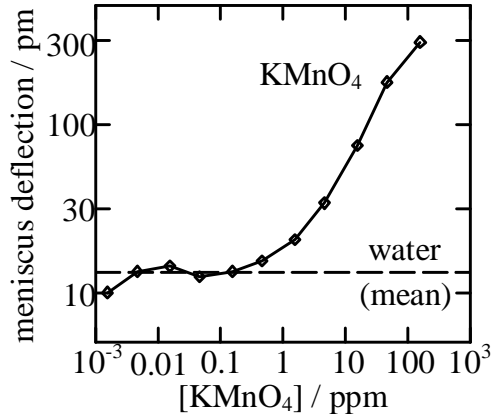

(a)

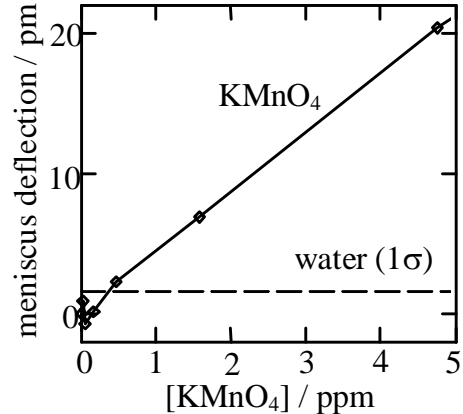

(b)

Figure 7. Photothermal rms meniscus deflection excited by a $678 \mathrm{~nm}$ laser diode. (a) Original data (b)

The same data following subtraction of the mean measurement for water.

Subtraction of the mean signal due to water gives a linear dependence on $\mathrm{KMnO}_{4}$ concentration at low concentrations above the noise level, as shown in Figure 7 (b). The minimum detectable concentration of $\mathrm{KMnO}_{4}(\mathrm{SNR}=1)$ is approximately $0.5 \mathrm{ppm}$. This corresponds to an additional absorption coefficient due to the $\mathrm{KMnO}_{4}$ of $4 \times 10^{-4} \mathrm{~cm}^{-1}$ (estimated using the diode array spectrometer) for which the absorbed power from the laser diode over $5 \mathrm{~cm}$ would be $6 \pm 1 \mu \mathrm{W}$ rms. At $678 \mathrm{~nm}$, the absorption due to water is expected to be $1.8 \times 10^{-3} \mathrm{~cm}^{-1}[3]$. The repeatability of the technique (one standard deviation) is therefore approximately $20 \%$ at $678 \mathrm{~nm}$.

\subsection{Photothermal detection of anthracene}

In the case of anthracene, the concentrations of the strongest solutions were calculated by comparing the measured absorption with the known literature value of the molar extinction coefficient, $2 \times 10^{5} \mathrm{~cm}^{-1} \mathrm{~mol}^{-1}$ litre at the $252 \mathrm{~nm}$ peak $^{[14]}$. The absorption coefficients of the weakest solutions were too low to be measured with the diode array spectrometer and so the concentrations were deduced from their dilution history. The absorption of anthracene at $254 \mathrm{~nm}$ was estimated to be approximately $75 \%$ of that at the peak wavelength.

Because the absorption coefficient of water at $254 \mathrm{~nm}$ is relatively high $\left(7 \times 10^{-3} \mathrm{~cm}^{-1}[15]\right)$, some additional discrimination was needed of the relatively small signal due to anthracene. Self-referencing was achieved by simultaneously measuring the photothermal signals due to $678 \mathrm{~nm}$ excitation (at $f_{2}=69 \mathrm{~Hz}$ ) and $254 \mathrm{~nm}$ excitation (at $f_{1}=115 \mathrm{~Hz}$ ). The additional absorption at $678 \mathrm{~nm}$ due to anthracene was negligible for all the solutions tested. The measured photothermal signals $(S)$ for low levels of absorption at $678 \mathrm{~nm}$ and $254 \mathrm{~nm}$ are then given by the following;

$$
\begin{aligned}
& S_{254}=\mathrm{A}\left[\alpha_{\mathrm{w}, 254}+\alpha_{\mathrm{a}, 254}\right] \frac{\mathrm{I}_{0,254}}{f_{1}} \\
& S_{678}=\mathrm{A}\left[\alpha_{\mathrm{w}, 678}\right] \frac{\mathrm{I}_{0,678}}{f_{2}}
\end{aligned}
$$

where A is a scale factor to be determined, which depends on (a) the cell responsivity and (b) the interference fringe height. The subscripts $\mathrm{w}$ and a denote water and anthracene, respectively. Taking blank reference measurements $(R)$ with deionised water gives a second pair of equations with a second scale factor B;

$$
\begin{aligned}
& R_{254}=\mathrm{B} \mathrm{I}_{0,254}\left[\alpha_{\mathrm{w}, 254}\right] \frac{\mathrm{I}_{0,254}}{f_{1}} \\
& R_{678}=\mathrm{B} \mathrm{I}_{0,678}\left[\alpha_{\mathrm{w}, 678}\right] \frac{\mathrm{I}_{0,678}}{f_{2}}
\end{aligned}
$$


Forming ratios of the $254 \mathrm{~nm}$ signals to the $678 \mathrm{~nm}$ signals, and subtracting the blank reference, then gives a measurement of the quantity of anthracene in solution, assuming that the level of absorption of the water at $678 \mathrm{~nm}$, relative emission intensities and modulation frequencies are all constant.

$$
\frac{S_{254}}{S_{678}}-\frac{R_{254}}{R_{678}}=\frac{\alpha_{\mathrm{a}, 254}}{\alpha_{\mathrm{w}, 678}} \frac{I_{0,254}}{I_{0,678}} \frac{f_{2}}{f_{1}}
$$

The ratio of the $254 \mathrm{~nm}$-excited to the $678 \mathrm{~nm}$-excited photothermal meniscus deflection signal, for each sample, has been plotted in Figure 8(a) against the estimated anthracene concentration in ppb by weight. Also shown is the mean of the photothermal signal ratios measured for a deionised water control (dashed line). Again, the same data are shown in Figure 8(b), following subtraction of the mean measured ratio for water.

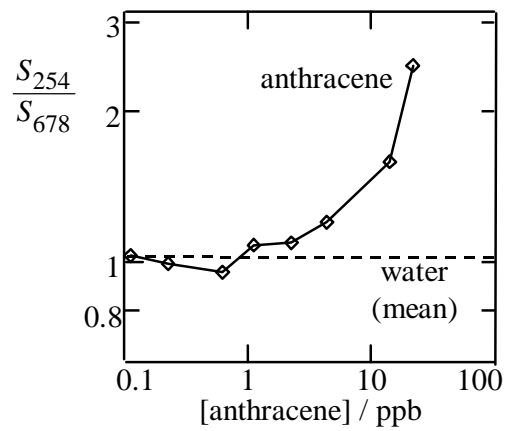

(a)

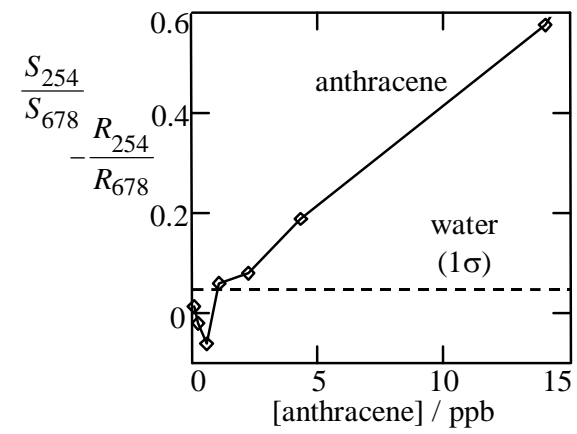

(b)

Figure 8. Photothermal results for anthracene and deionised water. (a) Ratio of $254 \mathrm{~nm}$-excited to $678 \mathrm{~nm}$-excited signals. (b) The same data following subtraction of the mean ratio for water.

These results indicate that our technique may be used to detect anthracene, in aqueous solution, at levels down to approximately $2 \mathrm{ppb}(\mathrm{SNR}=1)$. This corresponds to an absorption coefficient, due to anthracene alone, of approximately $1.7 \times 10^{-3} \mathrm{~cm}^{-1}$ at $254 \mathrm{~nm}$. The expected absorption coefficient of deionised water at $254 \mathrm{~nm}$ is $7 \times 10^{-3} \mathrm{~cm}^{-1}{ }^{[3]}$. Thus, the repeatability of the measurement (one standard deviation) is approximately $20 \%$ using the self-referencing technique.

\subsection{Investigation of alternative meniscus radii}

The apparatus was used to test the prediction of the Appendix, that the response to energy absorption, or indeed to any applied volume change within the cell, would be maximised by using a meniscus radius of $200 \mu \mathrm{m}$. The effect of the meniscus size on the magnitude of meniscus displacement was investigated using two different methods.

The most important measure of response was the magnitude of the photothermal signal with a known absorber. A solution of 500ppm $\mathrm{KMnO}_{4}$ was used, with an absorption coefficient of approximately $0.03 \mathrm{~cm}^{-1}$ at $678 \mathrm{~nm}$, absorbing approximately $30 \%$ of the incident light over the $5 \mathrm{~cm}$ path.

A second method involved using the cell PZT to apply standard volume changes to the cell, resulting in meniscus deflections that were proportional to the voltage applied to the PZT. This method had the advantage of producing a significantly larger meniscus deflection signal than the photothermal method. A large signal was needed because environmental noise resulted in excessively large low-frequency undulations of the largest meniscus tested. The cell PZT method correlated well with the photothermal measurements over a range of cell sensitivities, as shown in Figure 9. Therefore, this PZT method could reliably be used to establish the variation in system response with changing meniscus size. The responsivity 
of the cell, tested in this manner, has been expressed as the magnitude of the meniscus deflection per unit applied volt across the PZT.

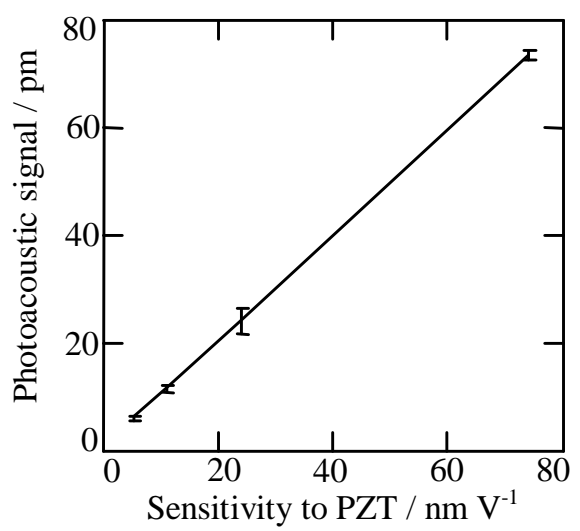

Figure 9. Correlation of two measures of system responsivity - the rms photothermal meniscus deflection, and the response of the system to applied sinusoidal voltages across the cell PZT. A straight line confirms that the latter may be used as a characteristic measure of system responsivity.

Measurements of system response, using the cell PZT method, are plotted in Figure 10 for menisci with different radii. Also plotted is the normalised dependence on meniscus size predicted by equation (12). The experimental data show a reasonable agreement with theory. Figure 10 implies that the optimum meniscus radius should lie between $200 \mu \mathrm{m}$ and $400 \mu \mathrm{m}$, which would be consistent with the cell being more compliant than has been assumed. However, our choice of a $200 \mu \mathrm{m}$ meniscus, from a limited number of possibilities, remains the best.

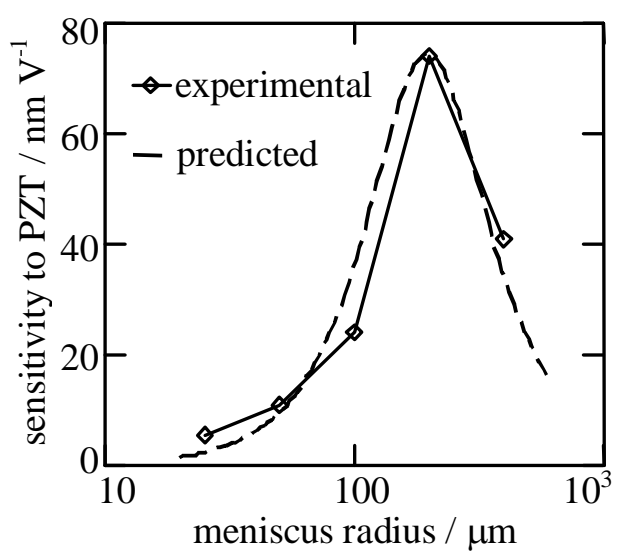

Figure 10. Optimum value of the meniscus radius determined by experiment, and the normalised prediction.

\section{Conclusion}

We have devised, constructed and tested a novel closed cell system for photothermal measurements of optical absorption in liquids. The system was tested with aqueous solutions of potassium permanganate, excited at $678 \mathrm{~nm}$ using a laser diode, and with anthracene, excited at $254 \mathrm{~nm}$ using a mercury discharge lamp. High sensitivity was achieved despite the use of low power, cw sources, potentially extending the applicability of photothermal / photoacoustic methods to low cost systems.

We overcame a number of constraints imposed by such light sources. The photothermal signal was small, and would be difficult to detect using traditional techniques involving piezoelectric microphones. Our 
novel pressure transducer involved a water meniscus acting as a highly compliant diaphragm, its deflection detected using fibre optic interferometry. The optimum radius for the meniscus gave it a compliance equal to the bulk compliance of the enclosed water. The signal to noise ratio of the photothermal measurements was enhanced by an acoustic suppression chamber, which reduced the level of ambient environmental acoustic noise. The limiting noise for the system was believed to be due to random fluctuations of the centre of the meniscus.

The ultimate sensitivity of the technique was limited by the background absorption of the deionised water solvent, expected to be $7 \times 10^{-3} \mathrm{~cm}^{-1}[15]$ at $254 \mathrm{~nm}$ and $1.8 \times 10^{-3} \mathrm{~cm}^{-1[3]}$ at $678 \mathrm{~nm}$. Subtracting a blank reference from the measured signal reduced the minimum detectable absorption coefficient, due to the analyte alone, to $4 \times 10^{-4} \mathrm{~cm}^{-1}$ at $678 \mathrm{~nm}(\mathrm{SNR}=1)$, corresponding to $0.5 \mathrm{ppm}$ potassium permanganate. For $254 \mathrm{~nm}$ measurements of anthracene absorption, a simultaneous reference signal was excited at a second wavelength not absorbed by anthracene. This compensated for the residual absorption of water to give a minimum detectable absorption coefficient of $1.7 \times 10^{-3} \mathrm{~cm}^{-1}$ at $254 \mathrm{~nm}$, corresponding to $2 \mathrm{ppb}$ anthracene. We do not appear to have encountered photoacoustic window noise, but it may have been hidden by the relatively high absorption of the water itself.

\section{Appendix - theory of operation}

We wish to establish the relationship between the deflection of the centre of the meniscus, $\Delta \mathrm{h}$, and the light energy absorbed by the aqueous analyte in the cell. The meniscus is shown in cross-section in Figure 11. The following assumptions have been made;

(i) The presence of trace chemicals in the water has a negligible effect on its thermal and mechanical properties, and those properties are unchanged (eg due to photothermal temperature increases) throughout the experiments.

(ii) A flat meniscus is used for detection purposes, with small deflections from this equilibrium.

(iii) The curvature of the meniscus is constant across the pinhole, ie surface forces dominate gravity and deflections are spherical.

(iv) The edge of the pinhole is perfectly sharp (zero radius of curvature).

(v) The pinhole can support any contact angle of fluid (real equilibrium contact angles would impose limits of applicability to the analysis).

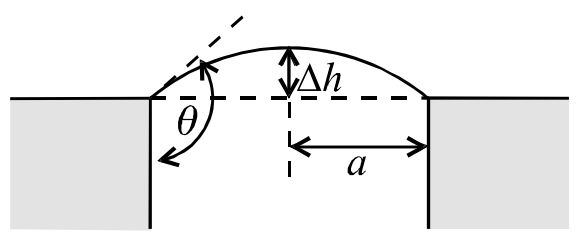

Figure 11. Cross-section of a liquid meniscus, at the edge of a small cylindrical orifice.

Firstly, the mechanical compliance of the cell is calculated, ie the change in volume caused by an internal pressure change. This is used to calculate the magnitude of the photothermal response, and the optimum value of the meniscus radius is found. Finally, the magnitude of the signal from the fibre optic interferometer is calculated. 


\section{Cell compliance}

The compliances $(\partial \mathrm{V} / \partial \mathrm{P})$ of different cell elements are additive, and may be considered one by one. The compliance of the water enclosed by the cell may be calculated from the definition of its bulk modulus $\kappa$ $\left(2 \times 10^{-9} \mathrm{~Pa}^{[16]}\right)$ and the internal volume of the cell $V_{\mathrm{c}}$.

$$
\left(\frac{\partial \mathrm{V}}{\partial \mathrm{P}}\right)_{\text {water }}=\frac{V_{c}}{\kappa}
$$

The compliance of the meniscus may be found as follows. The excess pressure across the surface of the liquid is given by the equation;

$$
\Delta P=\frac{2 \gamma \cos \theta}{a}
$$

where $\gamma$ is the surface tension of the fluid $\left(73 \times 10^{-3} \mathrm{~N} \mathrm{~m}^{-1}[17]\right)$. The meniscus compliance is lowest when $\theta$ $=\pi / 2$ and the meniscus is flat, which might at first appear surprising, because the excess pressure across an almost flat meniscus must be minimal. However, the displaced volume in this situation is also minimal, giving a low but non-zero value of the compliance. For small deflections from a flat surface, the meniscus compliance is approximately linear with respect to $\theta$, and the expression converges to the following;

$$
\left(\frac{\partial V}{\partial P}\right)_{\text {meniscus }}=\frac{\pi a^{4}}{8 \gamma}
$$

The total compliance of each of the cell windows and of the cell PZT, due to outward bending, may be calculated using deflection equations given by Timoshenko and Woinowsky-Krieger ${ }^{[18]}$, with the following result.

$$
\left(\frac{\partial V}{\partial P}\right)_{\text {window }}=\frac{\pi a_{w}^{6}}{16 Y t^{3}}\left(v^{2}-1\right)
$$

$a_{w}$ is the unsupported radius of the windows or PZT, $t$ is the thickness, $Y$ is the Young's modulus and $v$ the Poisson's ratio. The total combined compliance of $2 \mathrm{~mm}$ thick, $10 \mathrm{~mm}$ radius sapphire windows and a $0.48 \mathrm{~mm}$ thick, $3 \mathrm{~mm}$ radius wafer of PZT-5A was estimated to be less than $1 \%$ of the compliance of the water and the meniscus, and therefore could not significantly affect the magnitude of the photothermal response.

The total compliance of the cell is then the sum of all the significant individual compliances of the cell components, including the water contained within it, and is given by

$$
\frac{\partial V}{\partial P}=\frac{V_{c}}{\kappa}+\frac{\pi a^{4}}{8 \gamma}
$$

\section{Photothermal response}

When a cylindrical region of fluid lying in the optical path absorbs a small amount of light energy $\delta E$, its unconstrained volume increase would be,

$$
\delta V=\frac{\beta \delta E}{C_{p} \rho}
$$

where $\beta$ is the volume thermal expansion coefficient of the fluid $\left(207 \times 10^{-6} \mathrm{~K}^{-1}{ }^{[16]}\right), C_{p}$ is its specific heat capacity $\left(4.18 \mathrm{~J} \mathrm{~g}^{-1} \mathrm{~K}^{-1}[17]\right)$ and $\rho$ is its density. The pressure increase in a closed cell on absorption of energy $\delta \mathrm{E}$ is thus given by equation (10) and the total cell compliance.

It is proposed to measure the pressure increase in the cell by detecting the height change of the centre of the meniscus, $\Delta h$. The displacement of the flat meniscus from its mean position is a function of the excess pressure across it, which for small pressure changes approximates to the following linear expression; 


$$
\Delta h=\frac{a^{2} \Delta P}{4 \gamma}
$$

The response is a minimum for a flat meniscus, therefore we could increase the response by operating away from this point. However, by operating near the origin we should gain stability and linearity. Substitution gives the responsivity of meniscus deflection to absorbed energy.

$$
\frac{\partial \Delta h}{\partial E}=\frac{\beta a^{2}}{{ }^{4} C_{p} \rho \gamma} \frac{1}{\left(\frac{V_{c}}{\kappa}+\frac{\pi a^{4}}{8 \gamma}\right)}
$$

For any given cell size (which would preferably be as small as practically possible), there is an optimum value of $a$ which maximises the response. At this radius, the meniscus compliance is equal to that of the water enclosed by the cell.

$$
a_{\text {optimum }}=\left(\frac{8 \gamma V_{c}}{\kappa \pi}\right)^{\frac{1}{4}}
$$

For a cell containing pure water of volume $V_{c}=1.6 \times 10^{-5} \mathrm{~m}^{3}$, the optimum meniscus radius predicted by equation (13) is $200 \mu \mathrm{m}$. The overall responsivity to photothermal energy absorption then becomes;

$$
\frac{\partial \Delta h}{\partial E}=4 \times 10^{-4} \quad \mathrm{~m} \mathrm{~J}^{-1}
$$

\section{Detection of meniscus movement by fibre optic interferometry}

We used a low-finesse Fabry-Perot interferometer, formed by the centre of the water meniscus and the cleaved end of a single mode fibre. Santos et al have analysed such a cavity ${ }^{[19]}$, and concluded that for small reflectivities, a good approximation to a 2-beam or Fizeau interferometer can be made. Multiple reflections within the cavity are at a negligible level. If the amplitudes of the interfering beams are equal, the intensity of the fringes is given by;

$$
I=I_{0}\left\{1+K \cos \left(\frac{4 \pi d}{\lambda}\right)\right\}
$$

For an air-filled Fizeau cavity formed by silica (the cleaved fibre end) and water, the amplitudes of the two interfering beams will be slightly different, because the reflectivities of the two materials are dissimilar. However, in this case the resulting error is a small loss of fringe contrast rather than a change in fringe position, and so it may be neglected. For small signals, at the maximum voltage gradient (phase quadrature), the voltage signal $\mathrm{v}$ may be converted to a displacement $\mathrm{d}$ using the formula;

$$
\delta \mathrm{d}=\delta \mathrm{v} \cdot \frac{\lambda}{4 \pi A}
$$

The value of the scale factor $A$ may be determined by observation of the full-height fringes formed by large changes of optical phase.

\section{Acknowledgements}

This work was supported by North West Water Ltd and by the EPSRC / Department of Trade and Industry, through the UK Teaching Company Scheme. 


\section{References}

[1] Rosencwaig A 1980 Photoacoustics and photoacoustic spectroscopy (New York: Wiley)

[2] Pao Y-H 1977 Optoacoustic spectroscopy and detection (New York: Academic Press)

[3] Patel C K N and Tam A C 1981 Pulsed optoacoustic spectroscopy of condensed matter Rev. Mod. Phys. 53 517-550

[4] Hand D P, Hodgson P, Carolan T A, Quan K M, Mackenzie H A and Jones J D C 1993 Detection of photoacoustic waves in liquids by fibre-optic interferometry Opt. Commun. 104 1-6

[5] Hand D P, Freeborn S, Hodgson P, Carolan T A, Quan H A, Mackenzie H A and Jones J D C 1995 Optical fibre interferometry for photoacoustic spectroscopy in liquids Opt. Lett. 20 213-215

[6] Zahl H A and Golay M J E 1946 Pneumatic heat detector Rev. Sci. Instr. 17 511-515

[7] Golay M J E 1947 A pneumatic infra-red detector Rev.Sci. Instr. 18 357-362

[8] Schuman M 1965 Sensitivity of a selective pneumatic detector Appl. Opt. 41442-1445

[9] Moreau H, Hall J A, and Leaver V M 1957 Mercury-in-quartz thermometers for very high accuracy Rev. Sci. Instr. 34 147+

[10] Braslavsky S E, and Heibel G E 1992 Time-resolved photothermal and photoacoustic methods applied to photoinduced processes in solution Chem. Rev. 92 1381-1410

[11] Ulrich R, and Rashleigh S C 1980 Beam-to-fibre coupling with low standing-wave ratio Appl. Opt. 19 2453-2456

[12] Sigrist M W 1986 Laser generation of acoustic waves in liquids and gases J. Appl. Phys. 607 R83-R121

[13] Beltran F J, Ovejero G, Garcia-Araya J F and Rivas J1995 Oxidation of polynuclear aromatic hydrocarbons in water. 2. UV radiation and ozonation in the presence of UV radiation Indust. Eng. Chem. Res. 34 1607-1615

[14] Pavia L P, Lampman G M and Kriz G S Jr 1979 Introduction to Spectroscopy: A Guide for Students of Organic Chemistry (Harcourt Brace College Publishers)

[15] Hale G M and Querry M R 1973 Optical constants of water in the 200-nm to 200- $\mu$ m wavelength region Appl. Opt. 12 555-563

[16] Kaye G W C and Laby T H 1966 Tables of Physical and Chemical Constants (Longmans)

[17] Lide D R 1992 Handbook of Chemistry and Physics (CRC Press)

[18] Timoshenko S and Woinowsky-Krieger S 1959 Theory of plates and shells (McGraw-Hill)

[19] Santos J L, Leite A P and Jackson D A 1992 Optical fibre sensing with a low-finesse Fabry-Perot cavity Appl. Opt. 31 7361-7366 